\title{
Predictors of Mortality of Oncological Patients Admitted in the Pediatric ICU of a Tertiary Reference Hospital
}

\author{
Ynnaiana Navarro De Lima Santana Quintans ${ }^{1}$, Denise Bousfield Da Silva ${ }^{1,3}$, \\ Nilzete Liberato Bresolin ${ }^{2,3}$, Joana Sacheti Freitas ${ }^{1}$ \\ ${ }^{1}$ Department of Pediatric Oncology and Hematology, Joana de Gusmão Children's Hospital, Florianópolis, Brazil \\ ${ }^{2}$ Department of Pediatric Nephrology, Joana de Gusmão Children's Hospital, Florianópolis, Brazil \\ ${ }^{3}$ Department of Pediatrics, Federal University of Santa Catarina, Florianópolis, Brazil
}

Email address:

ynnaiananavarro@gmail.com (Y. N. De L. S. Quintans), denisebousfield@uol.com.br (D. B. Da Silva), nilzete.bresolin@hotmail.com (N. L. Bresolin), joanasacheti@gmail.com (J. S. Freitas)

\section{To cite this article:}

Ynnaiana Navarro De Lima Santana Quintans, Denise Bousfield Da Silva, Nilzete Liberato Bresolin, Joana Sacheti Freitas. Predictors of Mortality of Oncological Patients Admitted in the Pediatric ICU of a Tertiary Reference Hospital. Journal of Cancer Treatment and Research. Vol. 9, No. 3, 2021, pp. 45-49. doi: 10.11648/j.jctr.20210903.11

Received: June 2, 2021; Accepted: June 22, 2021; Published: July 9, 2021

\begin{abstract}
Cancer in the childhood and adolescence age group is considered rare. Despite this, the incidence of new diagnoses is increasing every day, as well as an increase in the cure rate, which is due, among other reasons, to the intensification of cancer treatment. As a consequence, these patients end up needing intervention in intensive care units (ICU). When compared to other patients, the mortality rate of cancer patients was higher than the general mortality in the pediatric ICU. For this reason, it is important to identify prognostic factors that can guide the early admission of these patients to the ICU. This study evaluated several variables related to the cancer patient admitted to the ICU, correlating them with the clinical outcome. Some variables were identified that increased the risk of the patient presenting with death as outcome, namely the number of organic dysfunctions on admission, the use of cardiotoxic chemotherapy, the use of nephrotoxic medications and the number of interventions performed on the patient in the first hours in the ICU. It is hoped that with this article, teams that provide health care to pediatric cancer patients will be able to identify children and adolescents in need of ICU interventions earlier, thus increasing their survival rate.
\end{abstract}

Keywords: Pediatric Oncology, Pediatric ICU, Organic Dysfunctions, Cardiotoxic Chemotherapy, Nephrotoxic Medications

\section{Introduction}

The pediatric cancer is considered rare, compared to cancers in adults, and varies proportionally according to geographic and population characteristics. It has its own histological and clinical characteristics, often affecting the hematopoietic system and supporting tissues. [1] Leukemias constitute most pediatric malignancies, followed by tumors of the central nervous system and lymphomas. [2]

The mortality rate varies according to geographic patterns. In Brazil and developed countries, it is the main cause of death from illness among children and adolescents. [3]

While the incidence of cancer in pediatrics has been increasing by $0.6 \%$ per year since 1975 , the survival of these patients has increased significantly. In 1970 , about $58 \%$ of children with cancer survived five years or more. Currently, the survival rate is around $80 \%$, possibly due to the use of multicentric treatment protocols, combined with improved diagnosis, the development of target therapies and the best quality of clinical support for critical patients. [2, 4]

The intensification of cancer treatment has led to an increasing number of complications resulting from therapeutic toxicity and immunodeficiency related to the disease and treatment. These complications can be fatal and require admission to a pediatric intensive care unit (PICU). In general, one in three pediatric cancer patients will require intensive care, [5] which represent $3-4 \%$ of pediatric ICU patients. [6].

It was found in the current literature that the mortality rate of cancer patients was higher than the general mortality of 
the pediatric ICU. Studies show worse outcomes for patients with hematological tumors, previous history of bone marrow transplantation, as well as those who develop cardiovascular, respiratory and / or renal failure, or who need inotropic support. [8-11] On the other hand, it is observed that patients admitted at the beginning of the diagnosis have a better prognosis than those admitted during treatment. $[7,5]$

In this context, it is essential to identify prognostic factors that can better guide the clinical decision for the early admission of these patients to the ICU, as well as the consequently appropriate management, preventing and addressing the main complications. Early detection, based on clinical protocols and predictive factors, can make admission, and stay in the ICU more effective, as well as improving the survival of these patients. $[5,7,9,10]$

This study aims to determine the main epidemiological characteristics and complications of pediatric cancer patients admitted to the ICU of a referral center in Florianópolis over a period of 8 years, as well as to identify the prognostic factors related to the outcome. In addition, the present study aims to establish the main predictors of mortality in these patients, so that aspects and events that may interfere with the effectiveness of intensive care are pointed out.

\section{Materials and Method}

This is a descriptive, retrospective, observational, crosssectional study carried out in the pediatric ICU of Children's Hospital Joana de Gusmão. The medical records of patients with oncological diseases in the age group up to 15 incomplete years admitted to the pediatric ICU of Hospital Tertiary, from January 2012 to December 2019, were analyzed. Patients aged 15 years or older, admitted to the ICU at the time of postoperative and medical records with incomplete data records were excluded from the research.

The variables analyzed included age, sex, histological type of the primary malignant neoplasm, stage of the disease at the time of diagnosis, clinical situation on admission to the pediatric ICU (stratified at the beginning of the diagnosis, being treated with remission of the malignant neoplasm or not, relapse of the disease), cause of admission to the ICU, length of stay prior to admission to the ICU, length of stay in the ICU, organ failure (number) at the time of admission and during hospitalization, need for interventions (inotropic support, dialysis, mechanical ventilation, nutritional support), use of nephrotoxic drugs (antibiotics, antifungals, chemotherapy), use of cardiotoxic chemotherapeutic agents and alteration of the echocardiogram performed in the first 24 hours of admission to the ICU.

Organic dysfunctions were assessed and classified according to the concepts used by Piastra and collaborators [12], that is, cardiovascular (hypotension requiring medical intervention, pericardial effusion, pericarditis, myocarditis, acute lung edema, symptomatic hypertension); respiratory (dyspnea or need for $\mathrm{O} 2$ therapy); neurological (depression of level of consciousness, acute neurological deficit); digestive (pancreatitis, veno-occlusive syndrome, gastrointestinal bleeding, graft versus host digestive disease, liver dysfunction); renal (acute renal failure); hematological (disseminated intravascular coagulation, hemophagocytic syndrome, anemia with $\mathrm{Hb}<4 \mathrm{~g} / \mathrm{dL}$ ) or metabolic (tumor lysis syndrome, symptomatic hyponatremia, hyperkaliemia with potassium $>6 \mathrm{mmol} / \mathrm{L}$ ).

To assess severity and predict patient outcomes, the PIM2 score (Pediatric index of mortality) was used. PIM 2 was calculated according to the physical examination and laboratory changes identified in the first hour of each admission, using the algorithm available in the article by Slater and collaborators [13], which is applied to the electronic calculator available at:: https://sfar.org/scores2/pim22.php.

To classify kidney injury, a known predictor, the Kidney Disease Improving Global Guidelines (KDIGO 2012) was used. [14]

These data were stored in an Excel spreadsheet.

The data were recorded in an Excel software table 2. Data entry and analysis were performed using SPSS (Statistical Package for the Social Sciences), version 20.0. Categorical variables were described by frequencies and percentages and continuous variables were described by median and interquartile range, with the $\mathrm{p} 25$ and $\mathrm{p} 75$ values, due to nonnormal distribution. To assess the association between categorical variables, the chi-square test or Fischer's exact test was used. To compare quantitative variables with asymmetric distribution between groups, the Mann Whitney test was used.

The OR association measure with the respective confidence intervals $(95 \% \mathrm{CI})$ was used. For all analyzes, a significance level of $5 \%$ was considered.

\section{Results}

The study sample consisted of 152 ICU admissions, with some admissions related to the same patient.

Among the admissions, $42.8 \%$ were female and $57.2 \%$ male. Hematological tumors corresponded to $75 \%$ of the population, while solid tumors corresponded to $25 \%$. Regarding the clinical situation, $73 \%$ of the children were in the first line of treatment and $27 \%$ went to the ICU under treatment for recurrence of the malignancy.

The main cause of admission to the ICU was septic shock, corresponding to $66.4 \%$ of the events, followed by respiratory failure $(9.9 \%)$, cardiogenic shock $(2.6 \%)$, hypovolemic shock $(1.3 \%)$. The group identified as "Others" (19.7\%), included episodes of upper gastrointestinal bleeding, stroke, decreased level of consciousness, hyperleukocytosis, tumor lysis, intracranial hypertension, renal failure requiring dialysis, seizure disorder and syndrome superior vena cava.

The length of hospital stay prior to admission to the ICU had a median of 4 days (interquartile range $=1-10$ ). The length of stay in the ICU had a median of 4 days (interquartile range $=2-7$ ).

There was a significant difference in the evaluation of the variables that analyzed the number of organic disorders 
(cardiovascular, respiratory, neurological, digestive, renal, hematological, or metabolic) on admission to the ICU $(\mathrm{U}=1217, \mathrm{p}<0.001)$ and then during the patient's hospitalization. ICU $(\mathrm{U}=1193, \mathrm{p}<0.001)$. The medians for these variables were 2 (interquartile range $=1-1$ ) and 2 (interquartile range $=1-3$ ), respectively.

Another variable that also showed a significant difference in relation to the outcome was the number of interventions that the patient received in the first 24 hours of admission to the ICU. The interventions evaluated were initiation of vasoactive drugs, installation of mechanical ventilation, initiation of parenteral nutrition and dialysis. The median of interventions was 2 (interquartile range $=1$ - 2) and it presented $\mathrm{p}<0.001$.

Regarding the use of myocardiotoxic chemotherapy in the treatment before admission to the ICU, $67.1 \%$ of the population used it. Within the population that used myocardiotoxic chemotherapy, $60.5 \%$ had the outcome "high" and 39.3\% the outcome was "death". In the sample that did not use this chemotherapy, $86.8 \%$ had the outcome "discharge" and 13.2\% "death". This variable showed a significant difference with the outcome "death" $(p=0.003)$.
When assessing the number of nephrotoxic drugs used by the patient before admission to the ICU and the outcome of death, a significant difference was also found $(p=0.034)$. The median use of nephrotoxic drugs was 1 , with an interquartile range of $0-2$.

Regarding the echocardiogram, $24.3 \%$ of the children had a normal exam on admission to the ICU, $5.9 \%$ of the exams were normal, but they were already performed with the child using vasoactive drugs and $53.9 \%$ had alterations, being them dysfunction or insufficiency of the left ventricle, dilation of the left cavities, severe myocardial depression, myocarditis, total dilation of the cavities, presence of intracardiac thrombus, pericardial effusion, hyperdynamic heart and obstruction of the superior vena cava. In this variable, we had $15.8 \%$ of patients who did not undergo echocardiography in the first 24 hours of admission to the ICU.

As for the outcome, there were 102 discharges $(67.1 \%$ of the sample) and 50 deaths (32.9\% of the sample).

Some of these variables are shown in Table 1, demonstrating their correlation with the clinical outcome, death or hospital discharge.

Table 1. Correlation between variables and clinical outcome

\begin{tabular}{llll}
\hline Variables & Death & Hospital Discharge & $\boldsymbol{p}$ \\
\hline Length of stay prior to ICU (days) & $5(2-15,5)^{\mathrm{A}}$ & $3(1-8)^{\mathrm{A}}$ & $0,174^{\mathrm{C}}$ \\
Length of stay in the ICU (days) & $4(2-9,5)^{\mathrm{A}}$ & $4(2-7)^{\mathrm{A}}$ & $0,555^{\mathrm{C}}$ \\
Organ failure (number) at the time of admission & $2(2-3)^{\mathrm{A}}$ & $1(1-2)^{\mathrm{A}}$ & $0,000^{\mathrm{C}}$ \\
Organ failure (number) during hospitalization & $3(2-3)^{\mathrm{A}}$ & $2(1-2)^{\mathrm{A}}$ & $0,000^{\mathrm{C}}$ \\
Number of interventions at the admission & $2(2-3)^{\mathrm{A}}$ & $1(0-2)^{\mathrm{A}}$ & $0,000^{\mathrm{C}}$ \\
Number of nephrotoxic drugs & $2(0-2)^{\mathrm{A}}$ & $1(0-2)^{\mathrm{A}}$ & $0,034^{\mathrm{C}}$ \\
Use of cardiotoxic chemotherapeutic agents & $45(29,6 \%)^{\mathrm{B}}$ & $69(45,4 \%)^{\mathrm{B}}$ & $0,003^{\mathrm{D}}$ \\
\hline
\end{tabular}

Note: A: Data presented as median P50 (P25 - P75); B: Data presented in absolute and relative frequency. Statistical significance defined as p $\leq 0.05$. C: MannWhitney test; D: Chi Square Test

\section{Discussion}

This research is a retrospective study, 8 years old, to evaluate the prognostic factors of mortality in pediatric cancer patients, hospitalized in the ICU due to acute complications of the underlying disease or treatment.

When compared to solid tumors, hematological neoplasms showed more severe conditions, as well as a higher percentage of infectious conditions and a higher mortality rate. Hematological neoplasms included acute lymphoblastic leukemia, acute myeloid leukemia, myelomonocytic leukemia, biphenotypic leukemia, Hodgkin's lymphoma Hodgkin's and non-Hodgkin's lymphoma, accounted for $75 \%$ $(n=114)$ of the sample and $82 \%(n=41)$ of deaths. A possible explanation for this fact must stem from the immunodeficiency of the underlying disease itself, from the more aggressive treatment protocols that contain corticosteroids as part of its structure in some longer periods.

Another trend identified was the relationship between a greater number of days prior to admission to the ICU and the outcome of death. Piastra and collaborators confirmed this observation in their study and, for this reason, established criteria for admission to the pediatric ICU for children with oncohematological diseases, with a strong recommendation for the early referral of these patients to the ICU, especially in cases of sepsis. [12]

The study by Fausser et al. Evaluated the causes that may determine this delay in admission to the ICU, identifying the reduced number of beds in some services; the transfer itself can be a time of complication, especially for neutropenic patients; the teams of oncologists and nurses in these sectors are trained to provide a high level of care, as they witness many complications in their wards, many potentially reversible complications without intensive treatment; and family members resistance to transfer. [15]

In this study, it was demonstrated that the number of organic dysfunctions present at admission to the ICU, as well as the amount of dysfunctions at the patient's hospitalization, had an impact on their mortality. Typpo et al., in a multicenter study with a population of 62,285 admissions to pediatric ICUs, showed that patients who had a higher number of organ dysfunctions, especially when more than three, had considerably higher morbidity than those who had a dysfunction at admission. In addition, patients were more likely to have at least one impairment of functional status maintained at the time of discharge from the ICU. [16] 
In a cohort of children admitted to the pediatric ICU in the United Kingdom, $27.3 \%$ of the patients studied had impaired functional status six months after admission to the ICU. Zinter and collaborators, in their multicenter cohort, retrospectively evaluated 264,364 admissions of cancer patients to the ICU. The interventions that these patients received at admission were analyzed, namely mechanical ventilation, hemodialysis, initiation of vasoactive drugs, installation of central access and oxygenation by extracorporeal membrane [17]. As in the present study, they observed that each new necessary intervention significantly increased the risk of mortality.

When considering the number of nephrotoxic drugs that the patient used before admission to the ICU, a significant difference was observed between the number of drugs used and the outcome of death. Historical analyzes had already proven this association [18-20], with increased morbidity and mortality in these patients and with a greater chance of developing chronic kidney complications, this association being progressively greater as a new nephrotoxic drug is associated with the patient's prescription. McKamy and colleagues, in a retrospective study, identified an increased risk of acute kidney injury from 3 to 9 times, when vancomycin was used concomitantly with furosemide [21]. In a meta-analysis published in 2018, Luther and colleagues suggested that the combination of vancomycin with piperacillin-tazobactam increased the chances of acute kidney injury compared to the combination of vancomycin with cefepime or meropenem [22].

As is already known, despite disease-free survival rates have increased in childhood and adolescence, survivors are at increased risk compared to the general population of having long-term morbidity and mortality, mainly due to treatmentrelated cardiotoxicity [23]. Dalton and collaborators reported that, when cardiac dysfunction is associated with the use of inotropic drugs and respiratory failure, the mortality of pediatric cancer patients admitted to the ICU increases to 54 to $100 \%$ in relation to those who do not have this association. [24]

Regarding the mortality rate of these patients in the ICU, in a historical review of the last 20 years, it was found that the results ranged from $13-27 \%$. It is worth mentioning that all studies in this review were international, some including patients after bone marrow transplantation, where mortality is higher [8, 24-28].

\section{Conclusion}

The mortality found in the present study was $32.9 \%$ $(n=50)$, slightly higher than that observed in historical reviews. Possible explanations for the mortality rate identified in this study, may be associated with the impossibility of early transfer of patients to the ICU due to the reduced number of beds at the time, or due to the immediate non-recognition of some severity warning signs and symptoms by the health team of on duty at the hospital, with consequent late admission to the ICU.

This study had some limitations, such as the non- identification of a specific type of neoplasia correlated with the outcome of death, probably since the diseases were grouped into two groups (hematological $\mathrm{x}$ solid), due to the sample size. As a limitation of the database, we obtained an important percentage of patients who did not undergo echocardiography at admission. As a limitation of data collection, it was not recorded which intervention was performed on the patient on admission to the ICU, it was only counted numerically.

With the analysis of these risk markers, it is expected that these data can help guide intensivists and oncologists in stratifying risk for patients, which can be of value in advising families concerning to the objectives of care, especially in times of neutropenia. Additionally, the identification of these markers can assist in the allocation of resources in the pediatric ICU, besides to corroborating the need to increase the number of beds in these units, especially those that are a reference for patients undergoing cancer treatment. Another important effect of the data is to identify patients who can benefit from closer monitoring and earlier interventions.

\section{Appendix}

Table 2. Data collection instrument.

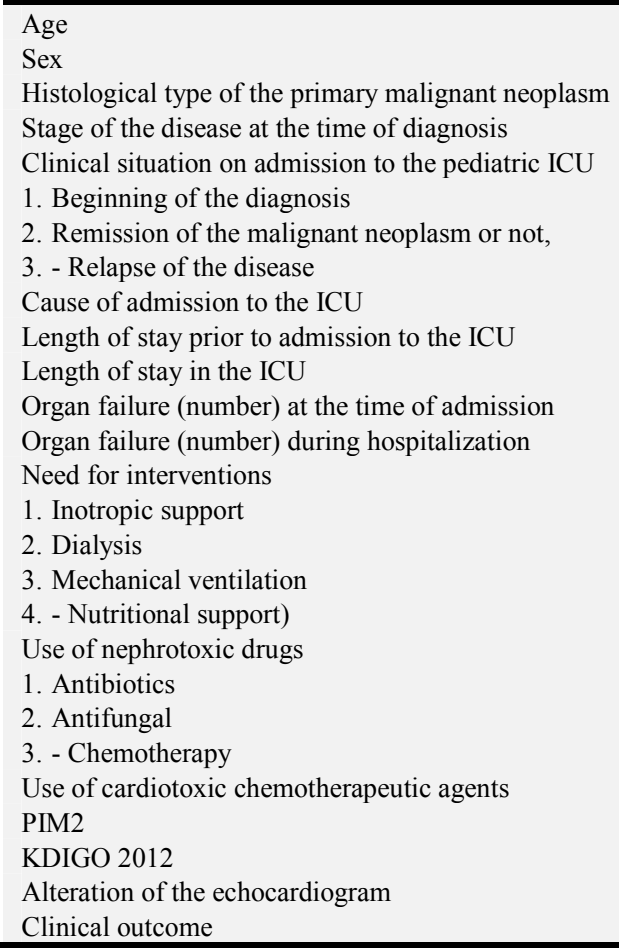

\section{References}

[1] Bustos, Q. J; Tumores cancerosos más frecuentes en pediatría: del oncólogo al pediatra. Pediatría. 1998; 14 (5): 251-8.

[2] Dorak, M. T.; Childhood Cancer Epidemiology. Mehmet Tevfik DORAK's Website. Avaible at: $<$ http://www.dorak.info/epi/ccepi.html $>$. Accessed in October 2017. 
[3] Ministério da Saúde. Instituto Nacional do Câncer. Incidência, mortalidade e morbidade hospitalar por câncer em crianças, adolescentes e adultos jovens no Brasil: Informações por registros de câncer e do sistema de mortalidade. Rio de Janeiro, 2016.

[4] American câncer society. Key Statistics for Childhood Cancers. Agosto, 2016. Avaible at: $<$ https://www.cancer.org/cancer/cancer-in-children/keystatistics.html>. Accessed in October 2017.

[5] Ali, A. M; Sayed, H. A; Elzembely, M. M.; The Outcome of Critically Ill Pediatric Cancer Patients Admitted to the Pediatric Intensive Care Unit in a Tertiary University Oncology Center in a Developing Country: A 5-Year Experience. J Pediatric Haemotol Oncol, 38: 355-359, 2016.

[6] Rosenman, M. B. et al. Hospital resource utilization in childhood cancer. J Pediatr Hematol Oncol, 27: 295-300, 2005.

[7] Heying, R. et al. Efficacy and outcome of intensive care in pediatric oncologic patients. Crit Care Med, 29: 2276-2280, 2001.

[8] Haase, R. et al. Management of Oncology Patients Admitted to the Paediatric Intensive Care Unit of a General Children 's Hospital - A Single Center Analysis. Klin Padiatr, 223: 142146, 2011.

[9] Abraham, B., R. et al. Predictors of outcome in pediatric intensive care units of children with malignancies. J Pediatric Haematol Oncol, 24: 23-6, 2002.

[10] Silvan, Y. et al. Outcome of oncology patients in the pediatric intensive care unit. Intens Care Med, 17: 11-5, 1990.

[11] Dursun, O., et al. Prognostic factors of pediatric cancer patients admitted to the pediatric intensive care unit. J Ped Hematol Oncol, 31: 481-4, 2009.

[12] Piastra, M, Fognani, G, Franceschi, A. Pediatric intensive care unit admission criteria for haemato-oncological patients: a basis for clinical guidelines implementation. Pediatr Rep., 3: 42-44, 2011.

[13] Slater, A., et al. PIM2: A Revised Version of the Paediatric Index of Mortality. Intensive care medicine, 29. 278-85, 2003.

[14] Kidney Disease: Improving Medical Outcomes (KDIGO) AKI Work Group. Kadigo clinical practice guideline for acute kidney injury. KIdyney Internacional Supplements 2012, v. 2, p. 1-138.

[15] Fausser, J. L. et al. Should We Pay Attention to the Delay Before Admission to a Pediatric Intensive Care Unit for Children With Cancer? Impact on 1-Month Mortality. A Report From the French Children's Oncology Study Group, GOCE. Journal of Pediatric Hematology/Oncology, 39: 5, 244-248, 2017.
[16] Typpo, K. V. et al. Day One MODS is Associated with Poor Functional Outcome and Mortality in the Pediatric Intensive Care Unit. Pediatr Crit Care Med, 10 (5): 562-570, 2009.

[17] Zinter, M. S., Pediatric cancer type predicts infection rate, need for critical care intervention, and mortality in the pediatric intensive care unit. Intensive Care Med, 40: 15361544, 2014.

[18] Gupta S, Sengar Gs, Meti Pk, Lahoti A, Beniwal M, Kumawat M. Acute kidney injury in pediatric intensive care unit: Incidence, risk factors, and outcome. Indian J Crit Care Med 2016; 20: 526-9.

[19] Bresolin N, Bianchini Ap, Hass Ca. Pediatric acute kidney injury assessed by pRIFLE as a prognostic factor in the intensive care unit. Pediatric Nephrol. 2013, 28 (3): 485-92.

[20] Freire KMS et al. Lesão renal aguda em crianças: incidência e fatores prognósticos em pacientes gravemente enfermos. Rev Bras Ter Intensiva. 2010; 22 (2): 166-74.

[21] Mckamy $\mathrm{S}$ et al. Incidence and risk factors influencing the development of vancomycin nephrotoxicity in children. J Pediatric. 2011; 158 (3): 422-6.

[22] Luther $M$ et al. Vancomycin plus Piperacillin/Tazobactam and Acute Kidney Injury in Adults: A Systematic Review and Meta-analysis. Critical Care Medicine. 2016, 46 (1): 1.

[23] Mertens Ac, Liu Q, Neglia Jp, Wasilewski K, Leisenring W, Armstrong Gt, et al. Cause-specific late mortality among 5year survivors of childhood cancer: the Childhood Cancer Survivor Study. J Natl Cancer Inst. 2008; 100 (19): 13681379.

[24] Dalton, H. J.; Slonim, A. D.; Pollack, M. M. Multicenter outcome of pediatric oncology patients requiring intensive care. Pediatric Hematol Oncol, 20: 643-9, 2003.

[25] MEYER S. et al. Assessing the risk of mortality in paediatric cancer patients admitted to the paediatric intensive care unit: a novel risk score? Eur J Pediatr 164: 563-567, 2-5.

[26] Owens C, Mannion D, O'marcaigh A, Waldron M, Butler K, O'meara A. Indications for admission, treatment and improved outcome of paediatric haematology/oncology patients admitted to a tertiary paediatric ICU. Ir J Med Sci 180: 85-89, 2011.

[27] $\mathrm{Ha}$ EJ et al. Early changes in SOFA score as a prognostic factor in pediatric oncology patients requiring mechanical ventilatory support. J Pediatr Hematol Oncol 32: e308-e313, 2010 .

[28] Gandhi, J.; Sangareddi, S.; Varadarajan, P. et al. Pediatric index of mortality 2 score as an outcome predictor in pediatric Intensive Care Unit in India. Indian J Crit Care Med, Sep-Oct, 17 (5): 288-291, 2013. 